\section{Response from the authors}

Dear Sir,

With reference to the comments from Dr. Parving regarding our study published in Diabetologia [1], I wish to offer the following explanations.

Regarding the cause of proteinuria in our patients, 20 $(59 \%)$ had diabetic retinopathy. Among the 14 patients who had no retinopathy only 5 were hypertensive, 2 had hypertension and diabetes diagnosed at the same time and 3 had hypertension diagnosed 3-9 years after the diagnosis of diabetes, around the time of presentation of proteinuria. The other 10 had known diabetes for a mean of 5.3 years. None of the 34 patients had other known causes of parenchymal or obstructive kidney diseases. We can thus assume that the great majority of the study patients had diabetic glomerulopathy.

At any rate, a benefit of cholesterol lowering therapy on renal function has also been reported in patients with non-diabetic nephrotic syndrome [2].

The aim of this study was to compare the effect on renal function of normalization of hypercholesterolaemia vs persistent hypercholesterolaemia, and not simply to compare the effects of lovastatin vs placebo on serum lipids and renal function. Thus the dose of lovastatin had to be increased until the target cholesterol level was achieved. There were therefore logistic difficulties in using the double-blind design. Anyway, although the physician was aware of the grouping, the control of glycaemia and hypertension was very similar between the two groups. Furthermore, the research nurse who carried out the glomerular filtration rate (GFR) studies and the laboratory technicians were not aware of the grouping of the study subjects and hence the conclusions regarding the changes in renal function could not have been affected by the study design.

We applied both intragroup and intergroup statistical tests which are both acceptable means of statistical analysis for these data. Although this study involves more patients and a much longer study period than previous studies addressing the benefit of cholesterol-lowering therapy on diabetic nephropathy $[3,4]$, the patient numbers and study duration were still

Corresponding author: Dr. K.S. L. Lam, Department of Medicine, The University of Hong Kong, Queen Mary Hospital, Hong Kong relatively insufficient for studying the progression of a chronic pathological process, for which the rate of deterioration is highly variable between patients though quite constant for a given individual. The above factors may explain why we could not demonstrate a statistically significant difference in GFR between the two groups at 24 months, although there was a trend towards a lower mean GFR in the placebo group. In the conclusion we have already conceded that our findings were preliminary and only supportive (not confirmative) of a potential benefit of cholesterol-lowering therapy on the progression of nephropathy in these patients.

The two studies referred to by Dr. Parving [3, 4] showed no benefir of simvastatin on renal function in IDDM (12 on treatment) and NIDDM ( 8 on treatment) patients after 12 and 36 weeks, respectively. These negative findings can be readily explained by the small numbers of patients and short study duration as explained above.

In conclusion, we believe that our conclusions, as presented in the published manuscript, are definitely valid. We also wish to thank the many workers in this field who have expressed interest in our preliminary findings. We certainly hope that longterm, multi-centre studies involving much larger numbers of patients can be carried out to address this controversial issue which is of potential therapeutic importance.

Yours sincerely,

K.S.L.Lam

\section{References}

1. Lam KS, Cheng IKP, Janus ED, Pang RWC (1995) Cholesterol-lowering therapy may retard the progression of diabetic nephropathy. Diabetologia 38: 604-609

2. Chan PCK, Robinson JD, Yang WC, Cheng IKP, Yeung HWD, Tsang MTS (1992) Lovastatin in glomerulonephritis patients with hyperlipidaemia and heavy proteinuria. Nephrol Dial Transplant 7: 93-99

3. Hommel E, Andersen P, Gall M-A et al. (1992) Plasma lipoproteins and renal function during simvastatin treatment in diabetic nephropathy. Diabetologia 35: 447-451

4. Nielsen S, Schmitz O, Moller N et al. (1993) Renal function and insulin sensitivity during simvastatin treatment in type 2 (non-insulin-dependent) diabetic patients with microalbuminuria. Diabetologia 36: 1079-1086

\section{The effect of cholesterol-lowering therapy on the progression of diabetic nephropathy is unproved}

\section{Dear Sir,}

In a recent paper Lam et al. [1] asserted that cholesterol-lowering therapy may retard the progression of diabetic nephropa-

Corresponding author: Dr. R.Bender, Heinrich-Heine-Universität Düsseldorf, Department of Metabolic Diseases and Nutrition, P.O. Box 101007, D-40001 Düsseldorf, Germany thy. However, the statistical analyses and the interpretation of the results give rise to the following criticism.

Firstly, the main hypothesis of any randomized clinical trial regarding the effect of an intervention can only be tested by means of between-group comparisons. However, the main conclusion of Lam et al. [1] was based upon within-group comparisons, which are principally invalid for investigating a treatment effect [2]. All tests between the two groups concerning the main outcome, namely simple $t$-tests for the glomerular filtration rate at each time point, yielded non-significant results. Hence, by means of the statistical analysis used by the authors, an effect of cholesterol-lowering therapy was not demonstrated.

Secondly, for within-group comparisons an invalid statistical method was used, namely two-way analysis of variance. 
Since the response curves are measured serially over time the resulting serial correlation has to be taken into account by using analysis of variance for repeated measurements [3]. However, as the within-group comparisons are unimportant for the main hypothesis one should focus attention on an appropriate analysis of the differences between the groups.

It may be that other more sophisticated methods for between-group comparisons have more power to show an effect of the intervention. There are three possibilities: 1) analysis of covariance with adjustment for relevant prognostic factors to reduce standard errors [4],2) analysis of variance for repeated measurements with one between-subject factor (group), one within-subject factor (time), and the corresponding interaction (group $\times$ time) to make use of the complete response curves [3], and 3) an analysis based upon adequate summary measures $[3,5]$. These methods would provide a more detailed analysis of the main study outcome. However, considering the large data variability, I presume that the sample size of the study is too small to detect a relevant difference between the groups.

In summary, the conclusion of Lam et al. [1] that cholesterol-lowering therapy may retard the progression of diabetic nephropathy is unproved because it is based upon inappropriate comparisons for which an invalid method was used. The pa- per is another example of the before-after fallacy, more examples of which can be found by Anderson [2].

Sincerely yours,

R. Bender

\section{References}

1. Lam KSL, Cheng IKP, Janus ED, Pang RWC (1995) Cholesterol-lowering therapy may retard the progression of diabetic nephropathy. Diabetologia 38: 604-609

2. Andersen B (1990) Methodological errors in medical research. Blackwell, Oxford London Edinburgh Boston Melbourne Paris Berlin Vienna

3. Crowder MJ, Hand DJ (1990) Analysis of repeated measures. Chapman and Hall, London New York Tokyo Melbourne Madras

4. Senn S (1994) Testing for baseline balance in clinical trials. Stat Med 13: 1715-1726

5. Matthews JNS, Altman DG, Campbell MJ, Royston P (1990) Analysis of serial measurements in medical research. BMJ 300: $230-235$

\section{Response from the authors}

Dear Sir,

We have read Dr. Bender's comments on the statistical methods employed in our paper on the beneficial effects of cholesterol-lowering therapy on the progression of diabetic nephropathy [1]. Taking into consideration the criticisms raised by Dr. Bender, we have employed alternative and more appropriate statistical methods to analyse the changes in glomerular filtration rate (GFR), serum creatinine and 24-h urine protein excretion in the two groups of patients studied. In each group, the incremental changes versus baseline values after 1 and 2 years of treatment, respectively, were analysed using the one-sample $t$-test (within group comparisons). The two-sample $t$-test was also used to compare the magnitude of such incremental changes between lovastatin- and placebo-treated patients at both time points (between group comparisons).

As shown in Table 1, a significant mean increment in serum creatinine was observed at both 1 year and 2 years in the placebo group, but only at 2 years in the lovastatin group. A trend towards an increase in 24-h urine protein excretion was seen in both groups at 2 years. A significant decrement in GFR was seen only in the placebo group and was observed at 2 years. These results from within group comparisons were similar to those we presented previously [1].

New findings were obtained from between group comparisons of the incremental changes in serum creatinine and 24-h urine protein excretion (Table 1). At 2 years, mean increments in serum creatinine and 24 -h urine protein were both smaller in the lovastatin group ( $p<0.05$ vs the placebo group).

Table 1. Effect of lovastatin on serum creatinine, 24-h urinary protein and glomerular filtration rate

\begin{tabular}{lllllll}
\hline & $\begin{array}{l}\text { Serum creatinine } \\
(\mu \mathrm{mol} / \mathrm{l})\end{array}$ & & $\begin{array}{l}\text { 24-h urine protein } \\
(\mathrm{g})\end{array}$ & & & \multicolumn{2}{l}{$\begin{array}{l}\text { Glomerular } \\
\text { filtration rate } \\
\left(\mathrm{ml} \cdot \mathrm{min}^{-1} \cdot 1.73 \mathrm{~m}^{-2}\right)\end{array}$} \\
\hline & 1-year & 2-year & 1-year & 2-year & 1-year & 2 -year \\
\hline Lovastatin $(n=16)$ & $2(-2,6)$ & $7(2,11)^{\mathrm{b}}$ & $0.005(-0.268,0.278)$ & $0.409(-0.008,0.826)^{\mathrm{a}}$ & $-2.95(-12.71,6.80)$ & $-2.03(-17.73,13.67)$ \\
Placebo $(n=18)$ & $6(1,11)^{\mathrm{b}}$ & $15(4,26)^{\mathrm{b}}$ & $0.291(-0.266,0.848)$ & $0.791(-0.060,1.641)^{\mathrm{a}}$ & $-2.76(-10.63,5.11)$ & $-10.85(-19.83,-1.87)^{\mathrm{b}}$ \\
$P$-value & 0.203 & 0.048 & 0.135 & 0.049 & 0.877 & 0.340 \\
$($-sample $t$-test) & & & & & & \\
\hline
\end{tabular}

Mean (95\% confidence intervals); ${ }^{a} p<0.1 ;{ }^{b} p<0.05,1$-sample $t$-test

Corresponding author: Dr. K. S. L. Lam, Dr. I. J. Lauder, Departments of Medicine, The University of Hong Kong, Hong Kong 
In conclusion, in this prospective 2-year study, cholesterollowering therapy was associated with significant reductions in the progressive rise in serum creatinine and urinary protein excretion in 16 NIDDM patients with nephropathy. In addition to the results previously presented [1], these observations provide further support for the potential beneficial effect of cholesterol-lowering therapy on the progression of nephropathy in patients with NIDDM.
Yours sincerely,

K.S.L.Lam, I.J.Lauder

\section{References}

1. Lam KSL, Cheng IKP, Janus ED, Pang RWC (1995) Cholesterol-lowering therapy may retard the progression of diabetic nephropathy. Diabetologia 38: 604-609

\section{Normal values of first-phase insulin response to intravenous glucose in healthy Italian children and adolescents}

Dear Sir,

Loss of first-phase insulin response (FPIR) to an intravenous glucose tolerance test (IVGTT) in first-degree relatives of diabetic patients with high levels of islet cell antibodies (ICA) and/or insulin autoantibodies, is highly predictive for insulindependent diabetes mellitus (IDDM) $[1,2]$. Normal values of FPIR for adult subjects have been established [1] but very few papers have been published on the paediatric population [3, 4]. The present study reports normal values of FPIR to IVGTT in a large paediatric population.

Working in close collaboration 21 Italian paediatric diabetes units tested 138 healthy subjects ( 47 females and 91 males) aged 3-20 years (mean: $10 \pm 3.7(\mathrm{SB})$ years), without endocrinopathy, short stature, obesity, family history of IDDM, history of drugs, history of hypoglycaemia or fasting plasma glucose more than $5.5 \mathrm{mmol} / \mathrm{l}$. In all subjects sex, age, weight, height, BMI and pubertal stage (according to Tanner) were recorded along with fasting plasma glucose, $\mathrm{HbA}_{1 \mathrm{c}}$, ICA and antibodies against glutamic acid decarboxylase $\left(\mathrm{GAD}_{65}\right)$. Subjects were divided into three groups on the basis of pubertal stage: Group 1 at stage I $(n=70)$, Group 2 at stages II-III $(n=41)$ and Group 3 at stages IV-V $(n=27)$. Before the study, the protocol was approved by the local institutional review board for human experimentation in each centre, and parents gave their written informed consent.

The procedure for the IVGTT followed the guidelines of the National Diabetes Data Group [5]. FPIR was expressed as the sum of the insulinaemia values at 1 and $3 \mathrm{~min}$. Fasting

Corresponding author: Dr. R. Lorini, Department of Pediatrics, IRCCS Policlinico San Matteo, P. le C. Golgi, 2, I-27100 Pavia, Italy

Acknowledgements. We would like to acknowledge the help of Professor C. Betterle (Istituto di Semeiotica Medica, Università di Padova), for ICA determination, Dr. A. Falorni (Department of Molecular Medicine, Laboratory for Molecular Immunology, Karolinska Institute, Stockholm) for detection of $\mathrm{GAD}_{65}$ antibodies, Dr. M. Ziveri and G. Nori (Istituto di Clinica Pediatrica, Università di Parma) for insulin assay, and Dr. E. Castoldi (Centro Elaborazione Dati, IRCCS Policlinico San Matteo, Pavia) for expert assistance in statistical analysis. insulinaemia/glycaemia ( $\mathrm{I} / \mathrm{G}$ ) ratio was also evaluated. Plasma glucose was measured by the glucose oxidase technique (glucose autoanalyzer, Beckman, Brea, California, USA), and $\mathrm{HbA}_{1 \mathrm{c}}$ levels using automatic HPLC (Bio-Rad, Brussels, Belgium). For determination of ICA, each center sent frozen serum from all subjects to Padova University [6]. For GAD $_{65}$ antibodies each center sent frozen serum to Karolinska Institute, Stockholm [7]. In each diabetes center blood samples for insulin assay were centrifuged at $4^{\circ} \mathrm{C}$ and then plasma was kept at $-20^{\circ} \mathrm{C}$, until analysis. The centers then sent all plasma samples for the measurement of insulinaemia to Parma University, for radioimmunoassay (Radim kit; Rome, Italy). The inter- and intra-assay coefficients of variation were $8.2 \%$ and $6.9 \%$ for low values and $7.7 \%$ and $6.0 \%$ for high values, respectively. Since the FPIRs during IVGTT were not normally distributed, the results are expressed as percentiles. Spearman's rank test was used to correlate fasting $\mathrm{I} / \mathrm{G}$ ratio with age and pubertal stage (groups 1 to 3 ).

A significant positive correlation was found between FPIR and chronological age $(p=0.046)$, in spite of the wide range of results observed. An increase in percentile values of FPIR was also found from Group 1 (prepubertal) through to Group 3 (pubertal) without significant differences between boys and girls (Table 1). Moreover, a significant positive relationship was observed between FPIR and pubertal stages ( $p=$ $0.0043)$ and BMI $(p=0.0052)$. Also fasting $\mathrm{I} / \mathrm{G}$ ratio showed a significant correlation with chronological age $(p=0.0015)$ and pubertal stages $(p=0.0015)$ in all subjects.

In our study we demonstrated that FPIR percentile values to an IVGTT increase from the younger children to the oldest group and from prepubertal to pubertal stages. Fasting I/G ratios showed a similar age- and pubertal-related pattern. Similar results have been reported by other authors who evaluated FPIR in ICA-negative first-degree relatives of IDDM patients $[8]$ and in normal subjects $[3,4]$. No significant difference was found between our boys and girls in Group 1 to Group 3 in terms of FPIR as Smith et al. [9] and Allen et al. [3] also reported, suggesting that gender does not affect insulinaemia changes during puberty. As children enter puberty at different chronologic ages, and their development is more related to their stage of puberty, we suggest that an accurate clinical staging of puberty rather than chronologic age may make more sense when interpreting IVGTT in young subjects at risk of IDDM followed-up throughout puberty. In fact, children may show rapid growth with pubertal development and thus a changing hormonal milieu that influences insulin sensitivity. Therefore, during the follow-up of children and adolescents at risk of IDDM it is very important to compare FPIR values taking into account pubertal stage instead of chronological age. 